\title{
Giant posterior gastric perforation in a 40 years old male: a case report
}

\author{
Raheel Hussan Naqvi*, Khushwant Singh, Rahul Singh
}

Department of Surgery, GMC, Jammu, Jammu and Kashmir, India

Received: 27 March 2021

Revised: 13 May 2021

Accepted: 17 May 2021

\author{
*Correspondence: \\ Dr. Raheel Hussan Naqvi, \\ E-mail: syedraheelnaqui@gmail.com
}

Copyright: (c) the author(s), publisher and licensee Medip Academy. This is an open-access article distributed under the terms of the Creative Commons Attribution Non-Commercial License, which permits unrestricted non-commercial use, distribution, and reproduction in any medium, provided the original work is properly cited.

\begin{abstract}
Posterior perforation of gastric ulcer is a rare clinical entity and has a propensity for delayed presentation and missed diagnosis. By virtue of their location, posterior gastric perforations may rupture into the retroperitoneum which leads to abscess formation and development of adhesions and consequently, leads to a delay in the onset of symptoms. Alternatively, ulcers located in the fundus or the body of the stomach may perforate into the lesser sac, which results in sealing off the perforation and less dramatic clinical signs and symptoms. This delay in presentation contributes to higher morbidity, higher infection rates and higher mortality. Thus, it is essential to have a high index of suspicion for this condition, particularly in patients with a protracted clinical presentation and classical risk factors so that timely, life-saving intervention is possible. We report a case of giant posterior gastric perforation with benign histopathology in a 40-years-old male. This case is being presented due to the rarity of this clinical entity and the relative paucity of literature on the same.
\end{abstract}

Keywords: Posterior perforation, Gastric perforation, Giant perforation, Spontaneous perforation, Emergency laparotomy

\section{INTRODUCTION}

Posterior perforation of a gastric ulcer is a rare condition. ${ }^{1}$ Majority of benign gastric ulcers lie along the lesser curvature of stomach. Only 5-8\% of these ulcers lie along the posterior wall of the body of stomach. ${ }^{2}$ The clinical presentation of patients is variable, depending upon the location of perforation. Ulcers located in the fundus or body of stomach may erode into the lesser sac which is less effective in sealing off the perforation. Gastric contents therefore, accumulate in the lesser sac and pass into the peritoneal cavity, resulting in generalized peritonitis. Posterior pyloric ulcers, on the other hand, are associated with rupture into retroperitoneum which may lead to abscess formation, adhesions and fibrosis. ${ }^{3}$ This explains why posterior gastric perforations are associated with delayed presentation, missed diagnoses and consequently, higher morbidity and mortality as compared to anterior perforations. $^{4}$
Helicobacter pylori, smoking and consumption of alcohol and other illicit drugs, use of NSAIDs and advanced age (>60 years) are important predisposing factors for the development of gastric ulcers. ${ }^{5}$ Chest radiography demonstrating pneumoperitoneum and computed tomography showing retroperitoneal air and/or fluid collection play an important role in the diagnosis of gastric perforation and determination of site of perforation. ${ }^{6}$ The standard of treatment for all patients with gastric perforation is emergency exploratory laparotomy. ${ }^{7} \mathrm{~A}$ biopsy of the ulcer margins is essential to establish the histological diagnosis. Repair of the perforation is done as a simple double layer closure with omental patch, which is often accompanied by a feeding jejunostomy. ${ }^{8}$

We present a case of a 40-years-old male with a spontaneous giant posterior gastric perforation with a benign histopathology. This case prompts discussion due to the rarity of its incidence in the general population. 


\section{CASE REPORT}

A 40 years old male with no known comorbidities presented to the emergency with chief complaints of pain abdomen, fever, absolute constipation and decreased urine output for the past 7-8 days. The pain was severe in intensity, insidious in onset, beginning in the peri-umblical region and gradually progressing to involve the entire abdomen. The patient was a smoker and had a history of cocaine abuse. On examination, the patient was febrile and had tachycardia. The pulse was feeble and the patient appeared sick-looking. Diffuse tenderness was present per abdomen examination and there were signs of full-blown peritonism. Hematological, biochemical and radiological investigations were sent. The complete blood count revealed severe anaemia and leucocytosis. Biochemistry was suggestive of prerenal azotemia. Chest radiography revealed pneumoperitoneum. Ultrasound abdomen showed free fluid in Morrison's space and pelvic cavity. After resuscitation, the patient was taken up for exploratory laparotomy. Around 31 of pus was drained from the peritoneal cavity and thick, dense adhesions were present between the gut loops and the parietal wall. A giant $4 \times 4 \mathrm{~cm}$ perforation was present on the posterior wall of stomach at the junction of the body and fundus along the greater curvature. The lesser sac had around $100 \mathrm{ml}$ of pus. Multiple biopsies ( $>10$ in number) of the ulcer margins were taken. The gastric perforation was closed in two layers and a feeding jejunostomy was done around $15 \mathrm{~cm}$ from the duodeno-jejunal junction.

Post-operatively, the patient developed wound infection followed by dehiscence for which diligent antiseptic dressing of the wound and subsequent secondary suturing was performed. The patient was discharged on antibiotics on the $15^{\text {th }}$ post-operative day and called for routine follow-up. The biopsy report revealed benign inflammatory changes.

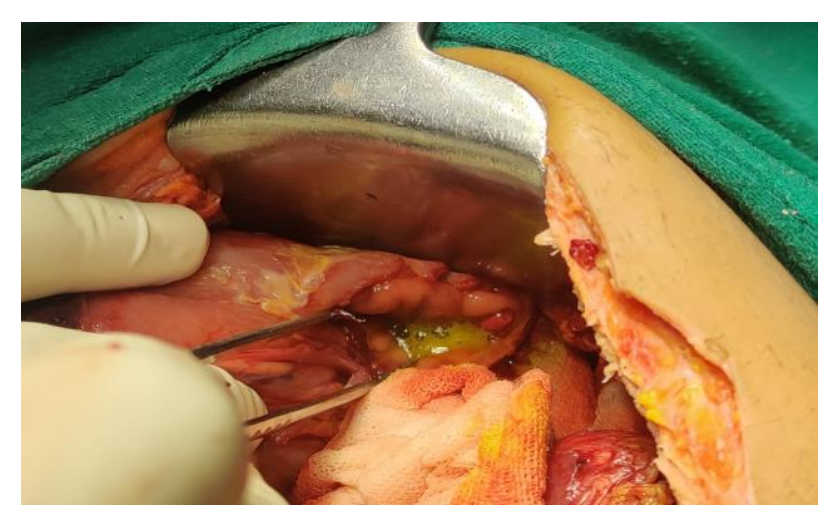

Figure 1: Giant posterior gastric perforation with Ryle's tube visualised in the stomach.

\section{DISCUSSION}

Posterior gastric perforation is a rare presentation and is associated with delayed presentation and high morbidity and mortality. As reported by Badawy and Wong et al, posterior gastric perforations had a high morbidity in patients to their rarity and the delay in clinical presentation. ${ }^{1,3,4}$ Risk factors include smoking and intake of illicit substances like cocaine as was observed in our patient. This is consistent with other studies reported in the literature such as the observations made by Uzzaman et al in his report. ${ }^{5,9}$ Pneumoperitoneum on chest radiography is an important diagnostic clue. ${ }^{6}$ Emergency exploratory laparotomy is the treatment of choice as reported in the literature by Kumar et al. ${ }^{7}$ Primary repair of the gastric perforation with an accompanying feeding jejunostomy is done in these patients as was seen in our patient. A biopsy of the ulcer margins is also imperative to establish the histopathological diagnosis. Due to delayed presentation and high morbidity, patients are often predisposed to wound infections. Hence, appropriate post-operative antibiotic cover and antiseptic dressing is essential for management.

\section{CONCLUSION}

A spontaneous, giant, posterior gastric perforation is a rare surgical emergency associated with high morbidity and mortality due to a relatively insidious onset of symptoms and frequently missed diagnoses. Emergency laparotomy and primary repair of the perforation is the standard of care as was carried out in this patient. Biopsies must also be taken to ascertain the histopathological diagnosis. It is essential to have a high index of suspicion in patients for this condition since early intervention is the key to facilitating recovery in them.

Funding: No funding sources Conflict of interest: None declared

Ethical approval: Not required

\section{REFERENCES}

1. Badawy AA. A large posterior perforation of gastric ulcer: A rare surgical emergency. Egyp J Surg. 2016;35:74-6.

2. Musgrove JE. Posterior penetrating gastric ulcer. Can Ned Assoc J. 1955;72:342-5.

3. Wong CH, Chow PK, Ong HS, Chan WH, Khin LW, Soo KC. Posterior perforation of peptic ulcers: presentation and outcome of an uncommon surgical emergency. Surgery. 2004;135(3):321-5.

4. Weston DWH, Perkiewicz M, Szczygiel B. Retroperitoneal extravasation from perforated duodenal ulcer. Br J Surg. 1988;75:878-9.

5. Gunshefski L, Flancbaum L, Brolin RE, Frankel A. Changing patterns in perforated peptic ulcer disease. Am Surg. 1990;56(4):270-4.

6. Wang SY, Cheng CT, Liao CH, Fu CY, Wong YC, Chen HW, et al. The relationship between computed tomography findings and the locations of perforated peptic ulcers: it may provide better information for gastrointestinal surgeons. Am J Surg. 2016;212(4):755-61. 
7. Kumar K, Pai D, Srinivasan K, Jagdish S, Ananthakrishnan N. Factors contributing to releak after surgical closure of perforated duodenal ulcer by Graham's Patch. Trop Gastroenterol. 2002;23(4):190-2.

8. Sarda DK, Garg PK. Study of incidence of peptic ulcer perforation in young adults: A hospital-based study. Int Arch Biomed Clinic Res. 2018;4:120-3.
9. Uzzaman MM, Alam A, Nair MS, Meleagros L. Gastric perforation in a cocaine user. Gastroenterol Hepatol. 2010;6(11):731-3.

Cite this article as: Naqvi RH, Singh K, Singh R.

Giant posterior gastric perforation in a 40-years-old male: a case report. Int Surg J 2021;8:1907-9. 